\title{
Causas políticas y consecuencias sociales de la corrupción ${ }^{1}$
}

\section{Joan Oriol Prats Cabrera}

Universitat Autònoma de Barcelona

Universitat Oberta de Catalunya

jpratscab@uuoc.edu

\section{Resumen}

Este artículo analiza empíricamente las causas políticas y las consecuencias sociales de la corrupción mediante datos de corte transversal para más de setenta países. En primer lugar, se analizan los determinantes políticos de la corrupción, prestando especial atención a los efectos de las relaciones entre poderes y los sistemas electorales sobre la arbitrariedad en la toma de decisiones públicas. En segundo lugar, se atiende a los efectos sociales de la corrupción; en concreto, a sus implicaciones para el gasto en educación y salud y los niveles de capital social o confianza interpersonal. Finalmente, se concluye resumiendo y realizando unas breves consideraciones generales sobre las estrategias futuras de lucha contra la corrupción.

Palabras clave: corrupción, control entre poderes, políticas sociales, capital social.

\section{Abstract. Political causes and social consequences of corruption}

This article analyzes empirically the causes and consequences of corruption using cross sectional data for more than seventy countries. First we examine the political determinants of corruption focusing on the effects of checks and balances and electoral systems on corruption. Second, we look at the consequences of corruption on public spending in health and education and social capital. Finally, we conclude summing up and making some general considerations for future anti-corruption programs.

Key words: corruption, political institutions, social policies, social capital.

\section{Sumario}

1. Determinantes políticos de la corrupción: rendición de cuentas vertical y horizontal

2. Las consecuencias sociales de la corrupción: bienes públicos y capital social
3. Conclusiones

Bibliografía

Anexo 1

1. El autor quiere agradecer a Josep Lluís Raymond Bara (Universidad Autónoma de Barcelona) su ayuda y colaboración. También se agradece el apoyo de la Fundación de Cajas de Ahorro a través de su programa de ayudas a la investigación y el apoyo económico del proyecto CICYT SEC2003-09773-C02-01/CPSO. La responsabilidad de lo escrito corresponde únicamente al autor. 


\section{Determinantes políticos de la corrupción: rendición de cuentas vertical y horizontal}

Varios artículos han analizado, de manera teórica y empírica, las causas políticas de la corrupción. Persson y Tabellini $(2001,2003)$ revisan estos estudios, modelan y prueban estadísticamente la influencia del tipo de régimen y del sistema electoral en los niveles percibidos de corrupción. Los trabajos de Treisman (2000a y b) abordan los efectos de la descentralización y el federalismo. En este trabajo, no obstante, se abordan únicamente los determinantes referidos a la rendición de cuentas vertical y horizontal. La rendición de cuentas vertical es la que se produce entre el gobierno y los ciudadanos a quienes representa. El principal mecanismo institucional por el que acaece este tipo de rendición de cuentas es el sistema electoral, mediante el que se escoge y se renueva a los gobernantes. La rendición de cuentas horizontal es la que se produce entre los poderes del Estado, es decir, entre el poder ejecutivo, el legislativo y el judicial. Ambos tipos garantizan la responsabilidad de los gobernantes y son indispensables en una democracia.

Persson y Tabellini (2001) analizan tres elementos del sistema electoral de manera teórica y empírica: el tipo de listas, el tamaño del distrito electoral y la fórmula electoral aplicada. En cuanto al tipo de listas, los autores sostienen que las que son abiertas promueven más la rendición de cuentas (accountability), puesto que obligan en mayor medida a cada uno de los políticos electos a responder ante un núcleo concreto de electores. Una mayor rendición de cuentas reduce la corrupción. En cuanto al tamaño del distrito electoral, consideran que, a medida que aumenta su extensión, más se reducen las barreras a la entrada de nuevos partidos y más aumenta la competencia entre éstos y, así, sus incentivos a mostrar una acción honesta de gobierno. Finalmente, en los sistemas bipartidistas, cuando la fórmula electoral es mayoritaria, se garantiza que el ganador obtenga al menos la mitad de los votos, lo que en principio hace que tenga que rendir cuentas ante un mayor número de personas.

Este trabajo enfatiza, no obstante, los efectos de los denominados «frenos y contrapesos» (checks and balances), más allá de sus implicaciones a través del tipo de régimen (democracia versus autocracia) y el sistema electoral. Los frenos y contrapesos se refieren a la relación entre poderes del Estado (ejecutivo, legislativo y judicial). Un mayor control de los actos del ejecutivo por parte del legislativo y del judicial reduce el nivel de arbitrariedad en las decisiones legislativas. Esto aumenta la credibilidad y reduce las oportunidades de realizar prácticas corruptas sin ser cogido. Cuando el ejecutivo no goza de una mayoría absoluta en el legislativo (y no tiene tantas facilidades para cambiar la legislación), la credibilidad percibida aumenta, porque no existe tanta capacidad de manipulación de las políticas ni de la administración ${ }^{2}$.

2. Este aspecto lo abordaron por primera vez Kydland y Prescott (1977) para el caso de la política monetaria y los problemas de inconsistencia temporal y credibilidad que todo gobierno confronta en su implementación. Así, estos autores proponen la delegación de la 
Existen pocos indicadores que den cuenta del nivel de «frenos y contrapesos» que provee el diseño constitucional. No obstante, desde hace ya tiempo, se vienen desarrollando indicadores que tienen por objetivo medir las variables institucionales o los niveles existentes de democracia, corrupción o favoritismo en las decisiones públicas. Varios think tanks y organismos internacionales y académicos generan, desde hace aún pocos años, datos que miden la calidad de las instituciones. Entre éstos figuran Freedom House, Fraser Institut, Transparencia Internacional y el Banco Mundial. La mayoría de estos indicadores son de carácter cualitativo, se basan en encuestas a expertos internacionales que muchas veces pueden estar sesgadas por la coyuntura internacional o la mala calidad de los datos de los países en vías de desarrollo. A nivel más académico, varios autores han desarrollado bases de datos en instituciones políticas (Database of Political Institutions - DPI) elaboradas por Beck y otros (2001), donde se recogen más de cincuenta variables políticas e institucionales, como, por ejemplo, la fragmentación del legislativo, el tipo de régimen (presidencialista, semipresidencialista o parlamentario), la polarización en el gobierno o el calor político del ejecutivo.

$\mathrm{El}$ indicador más preciso en términos de frenos y contrapesos o controles entre poderes del Estado es el realizado por Wiltold Henisz (2000), quien elabora un indicador en base a la cantidad de controles existentes y a la distribución de preferencias de los actores que actúan bajo dichos controles. Como existe una gran variedad de fórmulas concretas de control, la ciencia política comparada ha desarrollado el concepto de «actor estratégico» para referirse a aquel actor político (el gobierno, el ejecutivo o el judicial, pero también un partido político o un grupo de legisladores) que puede bloquear la agenda política (Tsebelis, 1990). El indicador de «frenos y contrapesos» (checks and balances) de Henisz tiene en cuenta el número de actores con capacidad de tomar decisiones e influir en la agenda política. La cantidad de actores estratégicos depende de las relaciones de dependencia que existen entre los distintos poderes. Cuando el ejecutivo tiene mayoría absoluta en el legislativo, éste deja de contar como un actor estratégico, y lo mismo sucede en el caso de un poder judicial completamente dependiente del ejecutivo en sus designaciones. Este indicador tiene además en cuenta el efecto que la fragmentación de los bloques políticos tiene sobre la capacidad de legislar. Por ejemplo, cuando el gobierno está en minoría en el legislativo, cuanto más fragmentada esté la oposición, menos dificultades tendrá para legislar y, a la inversa. Sin embargo, cuando el gobierno tiene mayoría, cuanto más frag-

política a un banco central independiente como forma de resolver el problema del compromiso que se deriva de los incentivos que tiene el gobierno para aprovecharse de la gestión monetaria en su propio beneficio. Sin embargo, para reducir el problema de credibilidad y control del poder ejecutivo en el diseño y la ejecución de otras políticas, son necesarios controles adicionales, como el que realizan el legislativo y el judicial, sobre todo en aquellas políticas que, como la industrial, la de sanidad y educación o la de pensiones, no son tan fácilmente delegables a organismos regulatorios independientes. 
mentado esté su bloque en el legislativo (más partidos estén incluidos en el gobierno), más dificultades tendrá para gobernar. Wiltold Henisz pondera estos efectos en su indicador ${ }^{3}$.

En el estudio estadístico que se presenta a continuación, se analiza hasta qué punto la corrupción (variable dependiente) viene determinada por el control entre poderes medido. Para medir la variable dependiente de este estudio, se utilizan dos indicadores de corrupción. El primero de ellos hace referencia a las percepciones de corrupción y está elaborado por Transparencia Internacional. El segundo está elaborado, desde el Instituto del Banco Mundial, por Kaufmann, Kraay y Zoido-Lobatón (1999) mediante una metodología de componentes no observados a partir de las encuestas realizadas por más de quince fuentes distintas. Siguiendo esta metodología, estos mismos autores elaboran indicadores para otras dimensiones institucionales clave: el estado de derecho y la estabilidad política.

En el cuadro 1 se muestran los efectos de las relaciones entre poderes sobre la corrupción, controlándolos por los niveles de renta, de educación, el origen colonial, de apertura y las regiones de mundo (véase el anexo 1 para la relación de fuentes). Se utilizan estas variables de control ya que la renta y la educación son factores que desincentivan y reducen la necesidad de apropiación de los recursos públicos en beneficio privado, a la vez que aumentan las capacidades de supervisión sobre los mismos. Estas variables han sido tradicionalmente incluidas en los estudios empíricos de la corrupción (Mauro, 1995; Treisman, 2000a). Las variables sobre el origen colonial pretenden tener en cuenta los efectos que, sobre la corrupción, ejercen los determinantes históricos comentados por la literatura, mientras que las regionales sirven para controlar e identificar las zonas del mundo especialmente retrasadas en este aspecto (Rodrik y otros, 2001; Acemoglu y otros, 2001).

Como se desprende del análisis (cuadro 1), los niveles de constricciones políticas o de control entre poderes reducen significativamente la corrupción, tanto si se utiliza el indicador elaborado por el Banco Mundial, como si se utiliza el realizado por Transparencia Internacional. La región del mundo donde la corrupción es significativamente mayor es en África, mientras que la influencia de la escolaridad y la apertura al exterior es muy pequeña (o insignificante) una vez se utilizan los controles pertinentes. También se observa que cuando se cambia la variable dependiente y se utiliza el estado de derecho, los resultados siguen siendo significativos, lo que resulta lógico, dada la elevada correlación que existe entre corrupción y estado de derecho $(0,95)$. Sin embargo, cuando la variable dependiente es la estabilidad política, se observa como el nivel de controles deja de ser significativo. Esto es consistente, puesto que la estabilidad política no depende directamente de la arbitrariedad existente en la toma de decisiones, sino de otros factores de carácter más exógeno y coyuntural, como el ciclo macroeconómico.

3. Para un análisis en mayor detalle del indicador, véase Henisz (2000). 
Cuadro 1. Corrupción y control entre poderes.

\begin{tabular}{|c|c|c|c|c|}
\hline \multirow[b]{2}{*}{$\begin{array}{l}\text { Variables } \\
\text { independientes }\end{array}$} & \multicolumn{4}{|c|}{ Variables dependientes } \\
\hline & $\begin{array}{l}\text { Índice } \\
\text { de corrupción }\end{array}$ & $\begin{array}{l}\text { Control de la } \\
\text { corrupción }\end{array}$ & $\begin{array}{l}\text { Estado } \\
\text { de derecho }\end{array}$ & $\begin{array}{l}\text { Estabilidad } \\
\text { política }\end{array}$ \\
\hline $\begin{array}{l}\text { Constricciones } \\
\text { políticas }\end{array}$ & $\begin{array}{l}-1,85^{* * *} \\
(1,76)\end{array}$ & $\begin{array}{l}0,59^{*} \\
(2,31) \\
\end{array}$ & $\begin{array}{l}1,63^{*} \\
(3,73)\end{array}$ & $\begin{array}{l}0,50 \\
(1,50)\end{array}$ \\
\hline $\begin{array}{l}\text { Logaritmo del PIB } \\
\text { por habitante }\end{array}$ & $\begin{array}{l}-1,29^{*} \\
(-3,87)\end{array}$ & $\begin{array}{l}0,74^{*} \\
(7,6)\end{array}$ & $\begin{array}{l}0,36^{*} \\
(2,53)\end{array}$ & $\begin{array}{l}0,30^{*} \\
(2,21)\end{array}$ \\
\hline $\begin{array}{l}\text { Matriculación } \\
\text { en primaria }\end{array}$ & $\begin{array}{l}0,02 \\
(1,17)\end{array}$ & $\begin{array}{l}0,007^{*} \\
(2,4)\end{array}$ & $\begin{array}{l}0,003 \\
(0,12) \\
\end{array}$ & $\begin{array}{l}0,005 \\
(0,38)\end{array}$ \\
\hline Apertura comercial & $\begin{array}{l}-0,02^{*} \\
(-2,93)\end{array}$ & $\begin{array}{l}-0,003 \\
(-1,16)\end{array}$ & $\begin{array}{l}0,001 \\
(1,43)\end{array}$ & $\begin{array}{l}0,005^{*} \\
(2,23)\end{array}$ \\
\hline África & $\begin{array}{l}-1,49^{*} \\
(-2,38)\end{array}$ & & & $\begin{array}{l}-0,37^{*} \\
(-2,01)\end{array}$ \\
\hline Asia & $\begin{array}{l}-0,47 \\
(-0,72)\end{array}$ & & & $\begin{array}{l}-0,09 \\
(-0,46)\end{array}$ \\
\hline América Latina & $\begin{array}{l}-0,34 \\
(-0,66) \\
\end{array}$ & & & $\begin{array}{l}0,21 \\
(0,73) \\
\end{array}$ \\
\hline$\overline{\mathrm{OCDE}}$ & $\begin{array}{l}-1,87^{*} \\
(-3,42) \\
\end{array}$ & & & \\
\hline Colonia española & & $\begin{array}{l}0,22 \\
(-1,35)\end{array}$ & $\begin{array}{l}-1,08^{*} \\
(-4,41) \\
\end{array}$ & $\begin{array}{l}-0,37^{*} \\
(-2,01)\end{array}$ \\
\hline Colonia inglesa & & $\begin{array}{l}0,35 \\
(2,18)^{*}\end{array}$ & $\begin{array}{l}-0,40 \\
(-1,63)\end{array}$ & $\begin{array}{l}-0,09 \\
(-0,46)\end{array}$ \\
\hline Colonia francesa & & $\begin{array}{l}0,36 \\
(1,3) \\
\end{array}$ & $\begin{array}{l}0,10 \\
(0,24)\end{array}$ & $\begin{array}{l}0,21 \\
(0,73)\end{array}$ \\
\hline R-cuadrado & 0,81 & 0,79 & 0,70 & 0,59 \\
\hline $\begin{array}{l}\text { Número } \\
\text { de observaciones }\end{array}$ & 72 & 74 & 70 & 74 \\
\hline
\end{tabular}

Los asteriscos *, ** y *** indican niveles de significación del 99, del 95 y del 90 por ciento, respectivamente. Estadísticos T entre paréntesis. Las celdas en blanco indican que esa variable independiente no ha sido incluida en la regresión. No se muestran los resultados para la constante.

Así pues, un mayor nivel de control entre poderes reduce la corrupción. Cuando el legislativo no cuenta con mayoría absoluta en el legislativo y no puede elegir discrecionalmente a los miembros de las altas dependencias judiciales, aumenta la credibilidad de las políticas públicas, puesto que es más difícil que un nuevo gobierno imponga un giro inesperado en la dirección de las políticas. De esta manera, el control horizontal entre los poderes políticos o una mayor fiscalización de los actos del gobierno están relacionados positivamente con el control de la corrupción. En aquellos regímenes políticos donde el ejecutivo 
Cuadro 2. Determinantes políticos de la corrupción: rendición de cuentas horizontal versus vertical.

\begin{tabular}{lll}
\hline & \multicolumn{2}{l}{ Variables dependientes } \\
\cline { 2 - 3 } & Control de la corrupción & Índice de corrupción \\
\hline Constricciones políticas & $0,81^{*}$ & $-2,43^{*}$ \\
& $(0,37)$ & $(1,03)$ \\
\hline Matriculados en primaria & $-0,01$ & 0,02 \\
& $(0,00)$ & $(0,01)$ \\
\hline Sistema mayoritario & $0,31^{*}$ & $-0,75^{* *}$ \\
& $(0,14)$ & $(0,37)$ \\
\hline Logaritmo del PIB & $0,87^{*}$ & $-2,03^{*}$ \\
& $(0,09)$ & $(0,26)$ \\
\hline R-cuadrado & 0,68 & 0,66 \\
\hline Observaciones & 71 & 71 \\
\hline
\end{tabular}

Los asteriscos * $\mathrm{y}^{* *}$ indican niveles de significación del 99 y del 95 por ciento, respectivamente. Errores estándar entre paréntesis. No se muestran los resultados para la constante.

no necesita negociar con otros partidos o controla totalmente el legislativo, resulta más fácil que unos pocos se apoderen de las decisiones públicas.

Como se puede observar en el cuadro 2, tanto el sistema electoral como el control entre poderes constituyen variables significativas para determinar los niveles de corrupción. Los resultados son consistentes con la literatura sobre la influencia de los sistemas electorales elaborada hasta el momento. Los sistemas con fórmula mayoritaria (aunque sólo muy ligeramente) reducen los niveles de corrupción, ya que responsabilizan al elegido ante un número mayor de ciudadanos (Persson y Tabellini, 2003). La influencia del control entre poderes es tanto o más importante que el tipo de sistema electoral.

\section{Las consecuencias sociales de la corrupción: bienes públicos y capital social}

Más allá de su impacto sobre el desarrollo económico, la corrupción tiene fuertes impactos sociales que aún han sido poco estudiados. En este apartado, se analiza cómo influye la corrupción sobre el nivel de bienes públicos y el capital social.

En primer lugar, cuando los poderes del estado están capturados por una elite o existen pautas clientelares que generan ineficiencia y arbitrariedad en la toma de decisiones, se reduce el gasto público efectivo en educación y salud. Tal y como se muestra en el cuadro 3, el gasto público en educación y salud por habitante respecto al PIB se reduce significativamente a medida que aumenta la corrupción. Esto es así incluso cuando se controla por el nivel de renta, el origen colonial y las distintas regiones del mundo, que en este caso sí se muestran significativas. Los resultados son consistentes cuando se estima el gasto 
Cuadro 3. Gasto en educación y salud y corrupción.

\begin{tabular}{|c|c|c|c|c|}
\hline \multirow[b]{2}{*}{$\begin{array}{l}\text { Variables } \\
\text { independientes }\end{array}$} & \multicolumn{4}{|l|}{ Variables dependientes } \\
\hline & $\begin{array}{l}\text { Gasto en educación } \\
\text { y salud } \\
\text { (por habitante) }\end{array}$ & $\begin{array}{l}\text { Gasto en educación } \\
\text { y salud } \\
\text { (por habitante) }\end{array}$ & $\begin{array}{l}\text { Gasto en educación } \\
\text { y salud } \\
(\% \text { del PIB })\end{array}$ & $\begin{array}{l}\text { Gasto en } \\
\text { educación y salud } \\
(\% \text { del PIB })\end{array}$ \\
\hline $\begin{array}{l}\text { Índice } \\
\text { de corrupción }\end{array}$ & $\begin{array}{l}-7,69^{*} \\
(-2,55) \\
\end{array}$ & $\begin{array}{l}-1,74^{*} \\
(-6,69) \\
\end{array}$ & $\begin{array}{l}-0,60^{*} \\
(-3,83) \\
\end{array}$ & $\begin{array}{l}1,63^{*} \\
(3,29) \\
\end{array}$ \\
\hline $\begin{array}{l}\text { Logaritmo del PIB } \\
\text { (por habitante) }\end{array}$ & $\begin{array}{l}2,18^{*} \\
(2,862)\end{array}$ & $\begin{array}{l}1,23^{*} \\
(1,972)\end{array}$ & $\begin{array}{l}0,35 \\
(0,775) \\
\end{array}$ & $\begin{array}{l}0,44 \\
(0,89)\end{array}$ \\
\hline África & $\begin{array}{l}-5,13^{*} \\
(-3,79) \\
\end{array}$ & & $\begin{array}{l}-4,39^{*} \\
(-5,23) \\
\end{array}$ & \\
\hline Asia & $\begin{array}{l}-5,33^{*} \\
(-3,69) \\
\end{array}$ & & $\begin{array}{l}-1,12 \\
(-1,24) \\
\end{array}$ & \\
\hline América Latina & $\begin{array}{l}-5,28^{*} \\
(-4,88) \\
\end{array}$ & & $\begin{array}{l}-2,34^{*} \\
(-3,44) \\
\end{array}$ & \\
\hline Colonia española & & $\begin{array}{l}-4,33^{*} \\
(-3,42) \\
\end{array}$ & & $\begin{array}{l}-2,73^{*} \\
(-3,78) \\
\end{array}$ \\
\hline Colonia inglesa & & $\begin{array}{l}-4,67^{*} \\
(-4,47) \\
\end{array}$ & & $\begin{array}{l}-2,03^{*} \\
(-2,75) \\
\end{array}$ \\
\hline Colonia francesa & & $\begin{array}{l}-3,68^{*} \\
(-3,20) \\
\end{array}$ & & $\begin{array}{l}-2,37^{*} \\
(-2,27) \\
\end{array}$ \\
\hline R-cuadrado & 0,70 & 0,82 & 0,73 & 0,63 \\
\hline Observaciones & 74 & 73 & 74 & 74 \\
\hline
\end{tabular}

El asterisco indica niveles de significatividad del 99\%. Estadísticos T entre paréntesis. No se muestran los resultados para la constante.

en educación y sanidad separadamente, tal y como se hace en el cuadro 4. En esta estimación, se introducen adicionalmente controles para el desempleo y la esperanza de vida para mejorar la robustez del modelo.

Un segundo efecto social clave de la corrupción es el que tiene sobre el capital social. Esto sucede sobre todo a través de sus efectos mediante «redes clientelares», que muchas veces se establecen en la Administración pública y operan a través de los cargos oficiales a favor de intereses particulares, normalmente vinculados a satisfacer a la clientela. Una red clientelar es una estructura de carácter jerárquico, donde cada elemento de la red responde ante un superior o patrón, a quien ha de satisfacer mediante criterios más personales que profesionales. Este fenómeno, conocido también como amiguismo o padrinazgo, da lugar a la ruptura de la separación que existe entre la esfera pública y la privada, lo cual permite el abuso de los poderes públicos y genera ineficiencias en los procesos de selección y gestión. Los efectos de esta telaraña de carácter jerárquico se traducen en una reducción de los niveles de capital social o confianza interpersonal entre los ciudadanos. 
Cuadro 4. Corrupción y gasto en educación y salud.

\begin{tabular}{|c|c|c|c|c|c|c|}
\hline \multirow[b]{2}{*}{$\begin{array}{l}\text { Variables } \\
\text { independientes }\end{array}$} & \multicolumn{6}{|c|}{ Variables dependientes } \\
\hline & $\begin{array}{l}\text { Gasto público } \\
\text { en educación } \\
\text { per cápita }\end{array}$ & $\begin{array}{l}\text { Gasto público } \\
\text { en educación } \\
\text { per cápita }\end{array}$ & $\begin{array}{l}\text { Gasto público } \\
\text { en educación } \\
\text { (\% del PIB) }\end{array}$ & $\begin{array}{l}\text { Gasto público } \\
\text { en sanidad } \\
\text { per cápita }\end{array}$ & $\begin{array}{l}\text { Gasto público } \\
\text { en sanidad } \\
\text { per cápita }\end{array}$ & $\begin{array}{l}\text { Gasto público } \\
\text { en sanidad } \\
\text { (\% del PIB) }\end{array}$ \\
\hline $\begin{array}{l}\text { Índice de corrupción } \\
\text { (transparencia } \\
\text { internacional) }\end{array}$ & $\begin{array}{l}-0,14^{*} \\
(-2,39)\end{array}$ & $\begin{array}{l}-0,10^{* *} \\
(-1,74)\end{array}$ & $\begin{array}{l}-0,07^{*} \\
(-2,59)\end{array}$ & $\begin{array}{l}-0,18^{*} \\
(-3,34)\end{array}$ & $\begin{array}{l}-0,11^{*} \\
(-2,33)\end{array}$ & $\begin{array}{l}-0,47 \\
(-4,90)\end{array}$ \\
\hline $\begin{array}{l}\text { PIB per cápita } \\
\text { (en logaritmos) }\end{array}$ & $\begin{array}{l}0,76^{*} \\
(5,217)\end{array}$ & $\begin{array}{l}0,72^{*} \\
(4,220)\end{array}$ & $\begin{array}{l}-0,01 \\
(-0,19)\end{array}$ & $\begin{array}{l}0,60 \\
(3,026)\end{array}$ & $\begin{array}{l}0,56^{*} \\
(2,759)\end{array}$ & $\begin{array}{l}-0,08 \\
(-0,23)\end{array}$ \\
\hline Desempleo & & & $\begin{array}{l}0,15^{*} \\
(2,471) \\
\end{array}$ & & & \\
\hline Esperanza de vida & & & & $\begin{array}{l}0,03 \\
(1,839)\end{array}$ & $\begin{array}{l}1,46 \\
(1,06)\end{array}$ & $\begin{array}{l}2,44 \\
(1,344)\end{array}$ \\
\hline Colonia inglesa & $\begin{array}{l}-0,55^{* *} \\
(-2,52)\end{array}$ & & $\begin{array}{l}-0,13 \\
(-1,30)\end{array}$ & $\begin{array}{l}-0,94^{*} \\
(-4,33) \\
\end{array}$ & & $\begin{array}{l}-1,73^{*} \\
(-4,67) \\
\end{array}$ \\
\hline Colonia francesa & $\begin{array}{l}-0,44 \\
(-1,38) \\
\end{array}$ & & $\begin{array}{l}-0,20 \\
(-0,94) \\
\end{array}$ & $\begin{array}{l}-0,87^{*} \\
(-2,81) \\
\end{array}$ & & $\begin{array}{l}-1,60^{*} \\
(-3,03) \\
\end{array}$ \\
\hline Colonia española & $\begin{array}{l}-0,81^{*} \\
(-3,60)\end{array}$ & & $\begin{array}{l}-0,25^{*} \\
(-2,30)\end{array}$ & $\begin{array}{l}-0,90^{*} \\
(-4,19)\end{array}$ & & $\begin{array}{l}-1,31^{*} \\
(-3,56) \\
\end{array}$ \\
\hline Asia & & $\begin{array}{l}-0,86^{*} \\
(-2,76)\end{array}$ & & & $\begin{array}{l}-1,80^{*} \\
(-6,70)\end{array}$ & \\
\hline África & & $\begin{array}{l}-0,49 \\
(-1,44) \\
\end{array}$ & & & $\begin{array}{l}-1,00^{*} \\
(-2,74) \\
\end{array}$ & \\
\hline América Latina & & $\begin{array}{l}-0,80 \\
(-3,16)^{*}\end{array}$ & & & $\begin{array}{l}-1,13^{*} \\
(-5,32)\end{array}$ & \\
\hline R-cuadrado & 0,80 & 0,78 & 0,44 & 0,87 & 0,79 & 0,76 \\
\hline Observaciones & 69 & 69 & 69 & 82 & 82 & 79 \\
\hline
\end{tabular}

Los asteriscos *, ** y *** indican niveles de significación del 99, del 95 y del 90 por ciento, respectivamente. Estadísticos $\mathrm{T}$ entre paréntesis. No se muestran los resultados para la constante.

La estructura jerárquica propia de las relaciones de padrinazgo o clientelares inhibe la relación horizontal entre los niveles inferiores de dicha estructura. La ayuda o la capacidad de prosperar pasa por la recompensa a la lealtad mostrada ante el patrón o el «caudillo». El bienestar de los ciudadanos no se relaciona entonces con su cooperación en la provisión de bienes colectivos (Maiz, 2002), sino que más bien al contrario, la estructura jerárquica propia de las redes de corrupción y la presencia misma de estas prácticas en la Administración pública reducen los niveles de confianza interpersonal y de capital social.

La importancia del capital social para el crecimiento la demuestran Zak y Knack (2001) mediante un modelo de equilibrio general y econométricamente utilizando los valores de confianza interpersonal de la Encuesta Mundial de 
Cuadro 5. Capital social y corrupción.

\begin{tabular}{|c|c|c|c|c|}
\hline \multirow{2}{*}{$\begin{array}{l}\text { Variables } \\
\text { independientes } \\
\text { Constante }\end{array}$} & \multicolumn{4}{|c|}{ Variable dependiente } \\
\hline & $\begin{array}{l}-3,21 \\
(-0,18)\end{array}$ & $\begin{array}{l}-31,8^{* * *} \\
(-1,79)\end{array}$ & $\begin{array}{l}-31,7 \\
(-1,64)\end{array}$ & $\begin{array}{l}-17,1 \\
(-0,41)\end{array}$ \\
\hline Control de la corrupción & $\begin{array}{l}8,51^{*} \\
(3,618)\end{array}$ & & $\begin{array}{l}10,8^{*} \\
(3,659)\end{array}$ & $\begin{array}{l}7,82^{*} \\
(2,512)\end{array}$ \\
\hline Índice de corrupción & & $\begin{array}{l}-4,78^{*} \\
(-4,44)\end{array}$ & & \\
\hline Logaritmo PIB por habitante & $\begin{array}{l}2,09 \\
(0,941)\end{array}$ & $\begin{array}{l}6,93^{*} \\
(3,616)\end{array}$ & $\begin{array}{l}6,60^{*} \\
(3,142)\end{array}$ & $\begin{array}{l}5,03 \\
(1,079)\end{array}$ \\
\hline Inflación & $\begin{array}{l}-0,01^{* *} \\
(-1,78)\end{array}$ & $\begin{array}{l}-0,01^{*} \\
(-2,44)\end{array}$ & $\begin{array}{l}-0,01^{* *} \\
(-1,79)\end{array}$ & \\
\hline Desigualdad & & $\begin{array}{l}2,67 \\
(0,840)\end{array}$ & $\begin{array}{l}3,65 \\
(1,086)\end{array}$ & \\
\hline $\begin{array}{l}\text { Gasto en sanidad } \\
\text { y educación (\% PIB) }\end{array}$ & $\begin{array}{l}1,42^{*} \\
(2,433)\end{array}$ & & & \\
\hline África & & & & $\begin{array}{l}-0,68 \\
(-0,09)\end{array}$ \\
\hline Asia & & & & $\begin{array}{l}-1,32 \\
(-0,15)\end{array}$ \\
\hline América Latina & & & & $\begin{array}{l}-8,81^{*} \\
(-2,04)\end{array}$ \\
\hline OCDE & & & & $\begin{array}{l}4,14 \\
(0,593)\end{array}$ \\
\hline R-cuadrado & 0,64 & 0,62 & 0,66 & 0,64 \\
\hline Observaciones & 48 & 48 & 36 & 36 \\
\hline
\end{tabular}

Los asteriscos ${ }^{*},{ }^{* *} \mathrm{y}^{* * *}$ indican niveles de significatividad del 99 , del 95 y del 90 por ciento, respectivamente. Estadísticos T entre paréntesis. No se muestran los resultados para la constante.

Valores (World Value Surveys) que conduce Ronald Inglehart. En esta encuesta, se pregunta a una muestra representativa de ciudadanos de más de cincuenta países cuál es su nivel de confianza interpersonal. Muchas veces se utiliza también cuantas probabilidades existen de que les devuelvan la cartera si la han perdido. Los valores distan desde un 10 por ciento en Perú, Brasil, Colombia o Turquía, a más de un 50 por ciento en Dinamarca, Suecia, China o Canadá.

En cuanto a los determinantes del capital social y sus efectos, se ha escrito bastante. Los trabajos realizados por Boix y Posner (1998) definen el capital social como la cooperación a largo plazo en la provisión de bienes públicos y privados a través de redes de confianza mutua que logran resolver el problema del oportunismo y mejorar la responsabilidad de los poderes públicos vía mayor supervisión y concienciación ciudadana. En el cuadro 5, vemos como 
la desigualdad y la propia corrupción influyen sobre los niveles de capital social. Resulta lógico pensar que cuanto mayor sea la corrupción, peores serán las percepciones y expectativas que los agentes tengan sobre el mercado y mayor será la desconfianza social. Éste constituiria un mecanismo similar al que afecta a la informalidad económica (Hellman y otros, 2000): las personas, al observar el funcionamiento del sector público, perciben también su propia realidad. Allí donde la clase política es clientelista y existe más soborno, los ciudadanos desconfían más los unos de los otros.

También en el cuadro 5 se puede observar como el nivel de gasto en bienes públicos, como son la educación y la sanidad, mejora el capital social existente. Esto puede deberse a las mayores capacidades que confiere un nivel más elevado de bienes públicos. Los ciudadanos, cuando disponen de servicios públicos que mejoran sus capacidades de emprender actividades sociales y económicas, tienden a mostrar mayores niveles de confianza interpersonal. Esto deja entrever un impacto indirecto del gasto público sobre el crecimiento a través de la generación de capital social.

\section{Conclusiones}

La corrupción es un fenómeno propio de todos los gobiernos que influye sobre los niveles de corrupción que explican los niveles de desarrollo, de gasto efectivo en bienes públicos y de capital social. Una mayor corrupción implica, en primer lugar, el aprovechamiento privado de las decisiones colectivas, lo que reduce el gasto en bienes y servicios de los que disfrutan la mayoría de los ciudadanos. A su vez, una mayor corrupción se identifica con una mayor ineficiencia y un uso de prácticas como soborno que generan desconfianza social. La corrupción opera también a través de relaciones jerárquicas de padrinazgo (fenómeno también conocido como clientelismo o amiguismo) que inhiben la creación de redes horizontales de confianza mutua o capital social.

En este trabajo se ha examinado la influencia del control entre poderes o de la capacidad del ejecutivo para legislar sin tomar en consideración al legislativo ni al judicial, y se ha mostrado cómo, cuando el ejecutivo no cuenta con una mayoría absoluta compacta en el legislativo y existe un poder judicial independiente, los niveles de corrupción son menores. Hasta el momento, la cooperación internacional ha dirigido acertadamente los esfuerzos hacia el funcionamiento de las instituciones electorales y aquéllas que, como el defensor del pueblo, garantizan el control vertical de la acción de gobierno (de los ciudadanos al gobierno). Sin embargo, también conviene tener en cuenta que gobiernos excesivamente poderosos ante el legislativo y no controlados por un poder judicial independiente que fiscalice sus actos legislativos y económicos mediante unos tribunales de justicia y de cuentas verdaderamente independientes y eficaces, suponen graves amenazas para el desarrollo. 


\section{Bibliografía}

Acemoglu, D.; Johnson. S.; Robinson, J. (2001). "The Colonial Origins of Comparative Development: An Empirical Investigation». American Economic Review, 91 (4): 1369-1401.

Alesina, A.; Reza, B.; Easterly, W. (1997). «Public Goods and Ethnic Divisions». Quarterly Journal of Economics, 114 (4): 1243-1284.

BECK, T. y otros (2001). «New tools in comparative political economy: The Database of Political Institutions». World Bank Economic Review, 15 (1): 165-176.

Borx, C.; Posner, D. (1998). "The Origins and Political Consequences of Social Capital». British Journal of Political Science, 28 (octubre): 686-693.

Hellman, J.; Jones, G.; Kaufmann, D. (2000). «Size the State, Size the Day. State Capture, Corruption and Influence in Transition». World Bank Policy Research Working Paper, 2444.

HeNISZ, W. (2000). «The Institutional Environment for Economic Growth». Economics and Politics, 12 (1): 1-31.

Kaufmann, D.; KraAY, A.; Zoido-Lobatón, P. (1999). "Aggregating Governance Indicators». World Bank Working Paper, 2195. Washington, DC.

KNACK, S.; KeEFER, P. (1995). «Institutions and Economic Performance: Cross-Country Tests Using Alternative Institutional Measures». Economics and Politics, 7 (3): 207227.

La Porta, R.; Lopez de Silanes, F.; Shleifer, A.; Vishny, R. (1997). «Legal Determinants of External Finance». NBER Working Paper, 5879. Cambridge MA.

- (1999). «The Quality Of Government». Journal of Law, Economics \& Organization, 15 (2): 222-83.

Lederman, D.; LOAYZA, N.; ReIS SOAReS, R. (2001). «Accountability and Corruption». World Bank Working Papers, 2708. Cambridge MA.

MAURO, P. (1995). "Corruption and Growth». Quarterly Journal of Economics, 106 (2): 681-711.

— (1997). «Why Worry About Corruption?». Economic Issues, 6. Fondo Monetario Internacional. Washington, DC.

MAIZ, Ramón (2002). «El clientelismo político como estructura de incentivos para la corrupción». Working Paper, 7/2002. Universidad Autónoma de Madrid.

MYERSON, R. (1993). «Effectiveness of electoral systems for reducing government corruption». Games and Economic Behaviour, 5: 118-132.

North, D. (1990). Institutions, Institutional Change and Economic Performance. Cambridge: Cambridge University Press.

Persson, T.; Tabellini, G.; Trebi, F. (2001). «Electoral Rules and Corruption». NBER Working Papers, 8151. Cambridge MA.

Persson, T.; Tabellini, G. (2003). The Economic Effect of Constitutions: What Do the Data Say? Cambridge Mass: The MIT Press.

Rodrik, D.; Subramanian, A.; TrebBi, F. (2002). «Institutions Rule: The Primacy of Institutions over Geography and Integration in Economic Development». NBER Working Paper, 9300. Cambridge MA.

Treisman, D. (2000a). «Decentralization and the Quality of Government». Artículo presentado en la Conferencia sobre descentralización fiscal del FMI. 20 y 21 de noviembre. Washington, DC.

- (2000b). «The Causes of Corruption: A Cross-National Study». Journal of Public Economics, 76 (3): 399-457. 
Tsebelis, G. (1990). Nested Games: Rational Choice in Comparative Politics. Berkeley: UC Press.

WACZIARG, R. (1996). Information to Create Colonization Dummies. Cambridge, MA. Mimeo.

ZAK, P.; KNACK, S. (2001). «Trust and growth». Economic Journal, 111: 295-321.

\section{Anexo 1}

Fuentes de los datos utilizados

Constricciones políticas: Witold Henisz (2000)

Sistema electoral mayoritario: Persson y Tabellini (2003)

Control de la corrupción: Kaufman y otros (2004)

Estado de derecho: Kaufman y otros (2004)

Estabilidad política: Kaufman y otros (2004)

Log PIB por habitante: World Development Indicators 2005 (Banco Mundial)

Matriculación en primaria: World Development Indicators 2005 (Banco Mundial)

Inflación: World Development Indicators 2005 (Banco Mundial)

Origen colonial: Wacziarg (1996)

Apertura comercial: Persson y Tabellini (2002)

Regiones del mundo: World Development Indicators 2005 (Banco Mundial)

Gasto público en educación World Development Indicators 2005 y salud por habitante: (Banco Mundial)

Gasto público en educación World Development Indicators 2005 y salud por habitante (\% PIB): $\quad$ (Banco Mundial) 